\title{
The Future of Dental Schools in Research Universities and Academic Health Centers
}

\author{
Laurie K. McCauley, DDS, MS, PhD
}

Abstract: As a profession, dentistry is at a point of discernible challenge as well as incredible opportunity in a landscape of evolving changes to health care, higher education, and evidence-based decision making. Respecting the past yet driving forward, a well-mapped future course is critical. Orchestrating this course in a collaborative manner is essential for the visibility, well-being, and potentially the existence of the dental profession. The research performed in dental institutions needs to be contemporary, aligned with biomedical science in general, and united with other disciplines. Dentistry is at risk of attrition in the quality of its research and discovery mission if participation with bioscience colleagues in the collaborative generation of new knowledge is underoptimized. A fundamental opportunity dentistry has is to contribute via its position in academic health centers. Rigorous research as to the impact of interprofessional education and collaborative care on population health outcomes provides significant potential for the dental profession to participate and/or lead such evidence-centered efforts. It is imperative that academic dental institutions are part of interdisciplinary and transdisciplinary organizations that move health care into its new day. Strategizing diversity by bringing together people who have different ways of seeing problems to share perspectives, heuristics, interpretations, technologies, and predictive models across disciplines will lead to impactful progress. Academic dental institutions are a natural part of an emphasis on translational research and acceleration of implementing new scientific discoveries. Dentistry needs to remain an essential and integrated component of higher education in the health professions; doing so necessitates deliberate, respectful, and committed change. This article was written as part of the project "Advancing Dental Education in the $21^{\text {st }}$ Century."

Dr. McCauley is Dean and William K. and May Anne Najjar Professor of Dentistry, Department of Periodontics and Oral Medicine, University of Michigan School of Dentistry and Professor, Department of Pathology, University of Michigan Medical School. Direct correspondence to Dr. Laurie K. McCauley, University of Michigan School of Dentistry, 1011 North University Avenue, Rm. 1234, Ann Arbor, MI 48109-1078; 734-763-3311; mccauley@umich.edu.

Keywords: research management, translational research, professional responsibility, economics of dental education, health care systems

Submitted for publication 1/13/17; accepted 3/9/17

doi: 10.21815/JDE.017.039

$\mathrm{T}$ The opportunity to impact human health through biologic discovery in dental and craniofacial research has never been greater. Our obligation to do this collaboratively is opportune and unreservedly essential for our future. Humans are not simple animals, and hence the solutions to health problems will not be simple answers. Systems biologic approaches will prevail, and new collaborative and team approaches are an imperative. We must respect and learn from the past, while evoking the responsible change necessary to secure a promising future. In 1995, the Institute of Medicine (IOM) report provided an imperative to dental education and science that has not only retained its importance but has undoubtedly become even more critical as we move forward: "Dental education must be scientifically based and undertaken in an environment in which the creation and acquisition of new scientific and clinical knowledge are valued and actively pursued. Research and scholarship are essential elements of university-based education and are critical to continued improvements in oral health."1
An illustrative clinical care example of our lack of collaborative approaches in solving multidisciplinary health problems initially surfaced in 2003, when reports arose of exposed necrotic bone in oral surgery patients who were taking anti-resorptive drugs called bisphosphonates. ${ }^{2}$ At the time, these cases were referred to as avascular necrosis and later termed osteonecrosis of the jaw (ONJ). The majority of patients described were cancer patients being treated for hypercalcemia, metastasis, and other comorbidities. These patients were taking medications other than the bisphosphonates, but the use of IV bisphosphonates was a commonality. In 2016, the precise etiology of this condition is as yet unclear. Over the past 15 years, there have been nearly 2,000 publications on this topic with fewer published in the dental literature (approximately 39\%) versus $60 \%$ published in the medical literature. In position papers and guidelines constructed over these years, there has been a consistent call for dentists to work with primary care physicians, endocrinologists, and oncologists for the clinical care of these patients. Yet 
there has been relatively minimal call for dentistscientists to work collaboratively with immunologists, cancer researchers, and microbiologists, for example, to discern the underlying etiology of this condition and virtually no evidence available for scientific-based guidelines for care.

As of July 2016, according to the National Institutes of Health (NIH) Research Portfolio Online Reporting Tools, there have been 11 projects with the words "osteonecrosis" and "jaw" in their titles. ${ }^{3}$ All have been funded by the National Institute of Dental and Craniofacial Research (NIDCR) and consist of three RO1s, four R21s, two RO3s, and two training mechanism grants. Of the $\mathrm{R}$ awards, the principal investigator is a DDS/DMD of five, MD of three, $\mathrm{DVM}$ of one, and $\mathrm{PhD}$ of one, suggesting a diversity of investigators, yet collaboration across disciplines is not well represented in the projects. Hence, 13 years after the initial case of ONJ, we still do not completely understand this multifaceted condition, and our patient care is not at a level it could be if we worked collaboratively in research to tackle this concern. If this type of model persists into the future, patient care will suffer.

Another example underlying a call to action involves the emergence of new and/or little-known conditions that take the world by storm. Although the Zika virus was first isolated in 1947 and the first cases were reported in humans in 1954, symptomatic Zika virus infections were limited to small numbers of patients. ${ }^{4}$ The first major outbreak of Zika occurred in 2007 in the Federated States of Micronesia, where $73 \%$ of the population was affected while only $18 \%$ were symptomatic. ${ }^{5}$ Since that time, Zika has spread rapidly and into many countries. It surfaced widely in the press with reports in 2015 and 2016 of the craniofacial developmental aberration of microcephaly in babies born to mothers infected with Zika. Suddenly there is a critical need for knowledge as to the incidence, basic underlying virology, pathogenesis of the craniofacial disorders, and impact on other physiologic systems. The embryologic development of the head and neck region is an area of strong historical strength in dental research institutions and one that could and should be mobilized when conditions that impact the craniofacial region arise suddenly and impactfully. One never knows when the next disease, drug-induced side effect, or environmental impact will surface that would benefit from dedicated dental and craniofacial research expertise. Dentistry is in danger of further losing its place as a player in the biosciences and at risk of attrition in the quality of its research and discovery mission due to lack of participation in the collaborative generation of new knowledge. The research performed in dental institutions needs to be more contemporary, aligned with biomedical science in general, and more collaborative.

Sixty-two leading public and private research universities that constitute the Association of American Universities (AAU) are where $62 \%$ of the nation's academic research is performed. ${ }^{6}$ These AAU institutions also produce $70 \%$ of the nation's scientists, engineers, doctors, teachers, and other professionals. Of these, 42 have medical schools, yet only 19 have dental schools. That means that less than one third of the current 66 dental schools are co-located with research-intensive AAU institutions. A broader indicator is the Carnegie classification of R1: Doctoral Universities-highest research activity. ${ }^{7}$ Of the 115 universities with this classification, a third (38) have dental schools. Hence even using this more inclusive classification, nearly $40 \%$ of U.S. dental schools are not associated with universities that have significant research activity. This pattern clearly raises a concern for our future ability to maintain sufficient scholarly activity to sustain a positive impact in optimizing the oral health of our communities.

A review of NIH funding to 56 academic dental institutions found that, of the top half of institutions receiving extramural support for research, more than $60 \%$ were located in AAU institutions - whereas, of the lower half, only $14 \%$ of these institutions were affiliated with an AAU university. ${ }^{8}$ Research universities as reflected by AAU membership are the hub of where new knowledge is produced. Other colleges and universities that do not significantly engage in research are primarily consumers and purveyors of the information generated at the research universities. Hence, the 19 dental schools located in AAU universities and, in particular, the 17 that are in the top half of NIH funding ranks are bearing the obligation for our profession to lead change based on solid and credible evidence that will improve patient care. Research-intensive universities are orchestrating grand challenges in which a team of scientists is put to task on a vexing problem and the diversity of team members predicts the success in the outcomes. Will dentistry be part of the grand challenge teams or be satisfied to focus on incremental technical progress? Future decisions regarding health in our society will be increasingly based on evidence. Will we be satisfied to sit on the sidelines and allow someone else to generate the evidence? This article was written as 
part of the project "Advancing Dental Education in the $21^{\text {st }}$ Century."

\section{Interdisciplinary and Transdisciplinary Collaboration in the Health Sciences}

A fundamental opportunity dentistry has as a profession is to contribute via its position in academic health centers. Academic health centers are defined as having a minimum of three components: a university-associated medical school, at least one other health professions school/program, and an owned or affiliated hospital. ${ }^{9}$ Nearly $40 \%$ of the academic health centers that are members of the Association of Academic Health Centers (AAHC) have dental schools, so that provides a robust opportunity for collaborative and interdisciplinary education, research, and patient care.

In 2015, the AAHC conducted a survey of leaders of academic health centers that included questions regarding the impact of health reform on their research missions and their institutional responses to those impacts. ${ }^{10}$ That study concluded that academic health centers will be successful "if they can function as organizations that align academics (teaching and research) with patient care and vice versa." Dentistry sorely needs to be part of the decision making and organizational discussion. Again, the IOM report in 1995 portended what has continued to be a leading concern in our profession in these words: "Dental education and dentistry are made vulnerable by their relative isolation from the broader university, from other health professions, and from the restructuring of health care delivery and financing that characterizes most of the health care system."1

Platforms of interprofessional education have emerged across the country and bear promise to progress to interprofessional care. According to the World Health Organization, "Interprofessional education occurs when students from two or more professions learn about, from, and with each other to enable effective collaboration and improve health outcomes. ... This is a key step in moving health systems from fragmentation to a position of strength."11 Rigorous research is needed to fill a gap of knowledge as to the impact of interprofessional education and interprofessional care (also known as collaborative care) on health outcomes for large-scale populations. ${ }^{12}$ There is a significant opportunity for the dental profession to participate and/or lead such an evidence-based collaborative effort.

The need for interdisciplinary and transdisciplinary organizations has emerged - yet not without roadblocks. ${ }^{13,14}$ Aspects of organizing the interdisciplinarity efforts can be taxing and present hurdles to discipline-centric fields like dentistry tends to be. While departments and schools value interdisciplinary efforts, they cling to traditional reward systems that promote individual accomplishments. Interdisciplinary work flows against decentralized financial models and often challenges grant management resources, facilities, and cost-sharing models. The $\mathrm{NIH}$ also often tends towards specific and narrow discipline-centric funding preferences, which further promulgates autonomous and siloistic institutional behavior. However, even the NIH is acknowledging and supporting resources for team-based research to address complex health issues. ${ }^{15}$ Academic dental institutions need to diversify their portfolios, focus on team science, and be integrated partners in biomedical research.

Over the past ten years (FY 2005-14), the NIH and NIDCR awarded extramural dollars to 56 academic dental institutions. ${ }^{8}$ During this time, the overall percentage of NIDCR funding to these institutions has remained at approximately $50 \%$ of the extramural award dollars; however, the total dollars to dental schools has decreased approximately $10 \%$. In addition, over this same period there was a 1.2-fold increase in numbers of proposals submitted to the NIH although a flat level of submissions to the NIDCR. Not only are we not moving forward, but by not growing, we are moving backwards. The mission of NIDCR is to improve dental, oral, and craniofacial health through research, research training, and the dissemination of health information. There is nothing explicit in this mission regarding supporting dental schools. It is the responsibility of our profession and our academic leaders to salvage the honor of our scientific obligation to generate new knowledge to improve the health of the public.

We must be part of interdisciplinary and transdisciplinary organizations that move health care into its new day, and strategizing the diversity that will underpin this success is crucial. Scott Page, a professor of complex systems at the University of Michigan, is a champion for diversity in problem-solving of all types. His work provides solid evidence that bringing groups of people together who have different 
ways of seeing a problem will be better and faster at problem-solving. He argues that people from different backgrounds have varying ways of looking at things, essentially different "tools." 16 A collective of such tools is more powerful than the ability of single individuals. His work brought forward mathematical modeling to support an equation: "Collective accuracy $=$ average accuracy + diversity." He posits that many of the problems we face in society (including disease) intersect with many traditional disciplines, and he strongly promotes interdisciplinary work. As he wrote, "These interdisciplinary efforts can be as crude as having people work together and communicate across disciplines, or they can result in new terminologies and frameworks. What's most important is sharing perspectives, heuristics, interpretations, and predictive models across disciplines. This approach will enable us to get off local peaks."

Dentistry has been atop its local peak for far too long, primarily afraid of venturing out at risk of losing its identity. Such fear and siloism will only restrict our ability to make impactful progress and render us vulnerable to demise. As Page urges, "We should recognize that a talented 'I' and a talented 'they' can become an even more talented 'we."'16

\section{Integration of the Biosciences}

Let us take inspiration from biology itself in all its complexity. Why would a simple approach solve a complex problem? Still, there are many barriers to integrating the biosciences. We are in a period of escalating regulatory burden that taxes our time, finances, and creativity. Our metrics of success are too frequently closely linked with funding versus impactful work. Hence, it is time we redefine our metrics for success. There are generational differences in our approach to science that challenge teams that span academic ranks. Our bioscience units lack common missions and goals. How often do we consider the culture of another unit when trying to align our research programs? Discrepant and/or decentralized budget models likely disincentivize collaborative work, and the decline in NIH funding challenges support for collaboration. The infrastructure including the physical location, age, and capacity of facilities on our campuses often presents barriers to integrating biomedical research groups. Time is often a restraint, with funding of projects at three to five years render- ing long-term impactful studies challenging. Finally, our electronic health records have opened new doors to providing large data sets with which to generate meaningful trends and ask prospective questions. However, the integration of dental electronic health records and medical electronic health records is virtually non-existent and an albatross when it comes to collaboration across health science fields. We must engage data scientists and informationists with our clinicians and clinical scientists.

In Wartman et al.'s survey of academic health centers regarding the impact of the Affordable Care Act on research, he found an "advantage of opportunities created by health reform to retool their research enterprise, with three-quarters of survey respondents increasing their focus on translational research, community-based research, and population health." ${ }^{10}$ Dental schools need to be a part of such an increased emphasis on translational research and acceleration of implementing new scientific discoveries. Respondents to Wartman et al.'s survey also widely supported academic health centers to "increase collaborations with industry groups and nonprofit funders of research to diversify research funding streams." Dentistry needs to better engage potential funders. Our pipeline of investigators capable of performing NIH-caliber research needs to be strengthened, so we can keep up with our peers in project submissions. Our clinical research enterprises need to partner with other health science units to assemble compelling proposals to community groups, foundations, and other federal agencies beyond the NIH. Well-established community-based, service-learning operations are present in most dental institutions and are unrivaled relative to other health professions. ${ }^{17,18}$ Three goals have been outlined for such service-learning opportunities: improving learning, promoting civic engagement, and strengthening communities. ${ }^{17}$ A natural fourth goal should be community-based research that is outcome-focused. These provide exciting new opportunities for our profession to shine. The time is now to retool, and academic dental institutions need to heed the warning signs that we could be losing ground if we do not.

In 2013, in a call to the broad health care community, Victor Dzau, president of the National Academy of Medicine, emphasized the need for stronger support for interdisciplinary work that is supported with core services: "we believe that academic health centers will need to increase the yields of research, accelerating the translation of results into practice and boosting their impact on medicine and 
health. Doing so will require establishing an effective 'discovery-to-care continuum' to facilitate more seamless translation, by creating structures integrating centers of clinical and translational research with offices of program management, regulatory affairs, education and training, biostatistics and biomedical informatics, and central biobanking, among others. Such structures can catalyze interdisciplinary collaborations and assemble resources into shared core services and facilities that offer natural economies of scale." ${ }^{19}$ It is virtually impossible in the current fiscal state of dental schools that we could pretend to be competitive in raising such structures in isolation of our bioscience colleagues. In collaboration with other health science units and beyond (engineering, public policy, and social sciences as examples), we must develop meaningful metrics of success apart from funding dollars or journal impact factors. Such metrics should demonstrate vital impact in communities and a spectrum of harnessing the etiology of diseases through to eradicating disease and preempting epidemics.

Another approach to integrate the biosciences is through the Learning Health System (LHS). The LHS is an integrated health system "in which progress in science, informatics, and care culture align to generate new knowledge as an ongoing, natural by-product of the care experience, and seamlessly refine and deliver best practices for continuous improvement in health and health care." ${ }^{20}$ A transformation is needed in which our electronic health records do more than simply record aspects of individual care, but also provide ongoing assessment of treatment effectiveness, trends in outcomes, and disease associations that can be used to collectively as well as individually inform decision making. A fundamental necessity for the success of such a system is the requirement for collaboration among various fields along with research, learning, and innovation that occur at the intersection of multiple and diverse disciplines. ${ }^{21}$

A key asset of academic health centers with prominent research missions is the collection and preservation of biologic samples and clinical data. In the era of big data, dental schools need to heed attention to the collection of clinical data that can become part of an LHS. Our electronic health records need to be orchestrated in a format that leverages the clinical data for outcome research and treatment effectiveness. The personalization of health care is well under way, yet dentistry has lagged behind our colleagues in other health professions. We need to operate on platforms that translate data through advanced analytics: platforms that engage patients through mobile devices, educational apps, and feedback systems to elevate health system performance via high-functioning interprofessional teams that include dentistry and provide such an individualized approach to patient care.

The current strained fiscal landscape of higher education in general, coupled with the opportunity for strengthening collaborative efforts, suggests that dental schools of the future will identify common aspects versus those that are essential to remain unique to dentistry. Faculty members may be shared across units located in research centers, and more cross-unit learning opportunities could exist for dental students. In order to orchestrate this, aspects of administration may need to be more centrally operated, while balancing the unique and defining aspects of the involved discipline. And, as Dr. William J. Gies emphasized back in 1926, let us not forget that we are a healing science, which necessitates that we remain contemporary in our approaches and embrace the most effective tools. ${ }^{22}$ Let us not forget that we are a learned profession requiring advanced learning and high principles, which necessitates the generation of new knowledge and the responsibility to apply research and discovery to improve patient care. Let us not forget we are an essential and integrated component of higher education in the health professions, which necessitates change that brings constant renewal.

\section{Editor's Disclosure}

This article is published in an online-only supplement to the Journal of Dental Education as part of a special project that was conducted independently of the American Dental Education Association (ADEA). Manuscripts for this supplement were reviewed by the project's directors and the coordinators of the project's sections and were assessed for general content and formatting by the editorial staff. Any opinions expressed are those of the authors and do not necessarily represent the Journal of Dental Education or ADEA.

\section{REFERENCES}

1. Field MJ, ed. Dental education at the crossroads: challenges and changes. An Institute of Medicine Report. Washington, DC: National Academies Press, 1995.

2. Migliorati CA. Bisphosphonates and oral cavity avascular bone necrosis. J Clin Oncol 2003;21:4253-4.

3. National Institutes of Health research portfolio online reporting tools. At: projectreporter.nih.gov/reporter.cfm. Accessed 13 Jan. 2017. 
4. Plourde AR, Bloch EM. A literature review of Zika virus. Emerg Infect Dis 2016;22:1185-92.

5. Duffy MR, Chen TH, Hancock WT, et al. Zika virus outbreak on Yap Island, Federated States of Micronesia. N Engl J Med 2009;360:2536-43.

6. Association of American Universities. At: www.aau.edu/. Accessed 13 Jan. 2017

7. Carnegie Classification of Institutions of Higher Education. At: carnegieclassifications.iu.edu/. Accessed 13 Jan. 2017.

8. Ferland CL, O'Hayre M, Knosp WM, et al. The NIH's funding to U.S. dental institutions from 2005 to 2014. J Dent Res, forthcoming.

9. Association of Academic Health Centers. At: www. aahcdc.org/. Accessed 13 Jan. 2017.

10. Wartman SA, Zhou Y, Knettel AJ. Health reform and academic health centers: commentary on an evolving paradigm. Acad Med 2015;90:1587-90.

11. World Health Organization. Framework for action on interprofessional education and collaborative practice. Geneva: World Health Organization, 2010.

12. Lutfiyya MN, Brandt BF, Cerra F. Reflections from the intersection of health professions education and clinical practice: the state of science of interprofessional education and collaborative practice. Acad Med 2016;91:766-71.

13. National Research Council. New directions in the sociology of aging. In: Waite LJ, Plewes TJ, eds. Roadblocks and bridges to transdisciplinary research: panel on new directions in social demography, social epidemiology, and the sociology of aging. Washington, DC: National Academies Press, 2013:56-79.
14. Hall KL, Feng AX, Moser RP, et al. Moving the science of team science forward: collaboration and creativity. Am J Prev Med 2008;35(2 Suppl):S243-9.

15. Hall KL, Olster DH, Stipelman BA, Vogel AL. News from NIH: resources for team-based research to more effectively address complex public health problems. Transl Behav Med 2012;2:373-5.

16. Page $S$. The difference: how the power of diversity creates better groups, firms, schools, and societies. Princeton: Princeton University Press, 2007.

17. Hood JG. Service-learning in dental education: meeting needs and challenges. J Dent Educ 2009;73(4):454-63.

18. Piskorowski WA, Fitzgerald M, Mastey J, Krell RE. Development of a sustainable community-based dental education program. J Dent Educ 2011;75(9):1038-43.

19. Dzau V, Cho A, ElLaissi W, et al. Transforming academic health centers for an uncertain future. N Engl J Med 2013;369:991-3.

20. Institute of Medicine. Digital infrastructure for the learning health system: the foundation for continuous improvement in health and health care. In: Grossman C, Powers B, McGinnis JM, eds. The foundation for continuous improvement in health and health care workshop series summary. Washington, DC: National Academies Press, 2011:1-311.

21. Friedman C, Rubin J, Brown J, et al. Toward a science of learning systems: a research agenda for the highfunctioning learning health system. J Am Med Inform Assoc 2015;22:43-50.

22. Gies WJ. Dental education in the United States and Canada. New York: Carnegie Foundation for the Advancement of Teaching, 1926. 